\title{
BMJ Open Neuroimaging biomarkers of psychogenic erectile dysfunction: protocol for a systematic review
}

\author{
Tao Yin, ${ }^{\oplus 1}$ Zhengjie Li, ${ }^{1,2}$ Jing Xiong, ${ }^{1}$ Lei Lan, ${ }^{1,2}$ Ruirui Sun, ${ }^{1,2}$ Feiqiang Ren, ${ }^{3,4}$ \\ Peihai Zhang ${ }^{4}$
}

To cite: Yin T, Li Z, Xiong J, et al. Neuroimaging biomarkers of psychogenic erectile dysfunction: protocol for a systematic review. BMJ Open 2019;9:e030061. doi:10.1136/ bmjopen-2019-030061

- Prepublication history for this paper is available online To view these files, please visit the journal online (http://dx.doi. org/10.1136/bmjopen-2019030061).

TY and ZL contributed equally.

Received 26 February 2019

Revised 29 July 2019

Accepted 31 July 2019

Check for updates

(c) Author(s) (or their employer(s)) 2019. Re-use permitted under CC BY-NC. No commercial re-use. See rights and permissions. Published by BMJ.

${ }^{1}$ Acupuncture and Tuina School/ The 3rd Teaching Hospital,

Chengdu University of Traditional Chinese Medicine, Chengdu, China

${ }^{2}$ Acupuncture-Brain Research Center, Chengdu University of Traditional Chinese Medicine, Chengdu, China

${ }^{3}$ Clinical Medicine School, Chengdu University of Traditional Chinese Medicine, Chengdu, China

${ }^{4}$ Urology and Andrology Department, Hospital of Chengdu University of Traditional Chinese Medicine, Chengdu, China

Correspondence to

Dr Peihai Zhang;

zhangpeihai@126.com

\section{ABSTRACT}

Introduction Erectile dysfunction (ED) is the most common male sexual disorder that severely impacts the sexual performance and quality of life of men. As the main subtype of $E D$, psychogenic $E D(p E D)$ has been demonstrated to be a genitourinary disease and also associated with alterations in both brain structure and function. However, the scattered neuroimaging evidence from individual studies has not yet been integrated, and the central pathological alterations associated with $\mathrm{pED}$ remain unclear. The objective of this systematic review is to integrate and assess the evidence of the impact of pED on brain structure and function.

Methods and analysis Five databases (PubMed, EMBASE, Web of Science, China Biology Medicine Database and China National Knowledge Infrastructure (CNKI)) will be systematically searched from inception to 1 October 2019 (the anticipated completion date of this review), with language restricted to English and Chinese. Studies focusing on the structural or functional alterations in patients with $\mathrm{pED}$ will be retrieved. The study selection process will follow the Preferred Reporting Items for Systematic Reviews and Meta-Analyses guideline and quality assessment will be conducted with a customised checklist. After data extraction, a qualitative review will be performed to synthesise the structural and functional brain alterations as well as the correlations between the altered cerebral structures and functions and the clinical characteristics of patients with $\mathrm{pED}$. If the collected data make it feasible, an activation likelihood estimation metaanalysis will also be launched.

Ethics and dissemination Ethical approval is not required as primary data will not be collected. This review will be published in a peer-reviewed journal and presented at conferences.

PROSPERO registration number CRD42019117206.

\section{INTRODUCTION}

Erectile dysfunction (ED) is the most common male sexual disorder. It is characterised by the persistent inability to attain or maintain an adequate erection to obtain satisfactory sexual intercourse regardless of the capability of ejaculation. ${ }^{1-3}$ According to epidemiological studies, approximately $37 \%$ of men over 70 years old and $11 \%$ of men over 30 years old suffer from this sexual

\section{Strengths and limitations of this study}

- This is the first systematic review and meta-analysis that integrates and assesses the central pathological characteristics of psychogenic erectile dysfunction.

- Qualitative and quantitative synthesis (activation likelihood estimation meta-analysis) will both be used in this study.

- A customised checklist is proposed to evaluate the quality of the included studies according to the purpose of this review.

- This review does not restrict the race, age, disease conditions of participants or preprocessing procedures of included studies, which will increase the heterogeneity of included studies and may increase the risk of bias of the review.

dysfunction. ${ }^{4}$ As a physical and psychosocial illness, ED not only impairs male sexual confidence and satisfaction but also severely impacts the quality of life $(\mathrm{QoL})^{56}$ and relationships $^{7}$ of patients and their partners. More importantly, ED has been increasingly regarded as an independent risk factor for cardiovascular diseases. ${ }^{8} 9$ According to its different causes, ${ }^{10}{ }^{11} \mathrm{ED}$ is subdivided into psychogenic ED (pED), organic ED and mixed ED. Different from organic ED, which has clear causes and pathological characteristics, pED is generally caused by uncertain psychological factors ${ }^{12} 13$ and lacks specific biomarkers.

\section{Rationale for review}

Penile erection is a complex physiological process modulated by the central nervous system and mediated by several neurotransmitters and neuropeptides. ${ }^{1415}$ A meta-analysis identified that penile erection was regulated by several cerebral regions; the activities of the insular cortex, claustrum, putamen and anterior midcingulate cortex were consistently positively correlated with male penile erection. ${ }^{16}$ With the close relationship between the brain and penile erection being widely 
accepted, using neuroimaging techniques to explore the central pathological characteristics of pED has attracted the attention of many researchers. ${ }^{17-22}$ In the process, some well-designed cognitive-behavioural models have been developed to further explain the neurobiological underpinning of abnormal behaviour in patients with ED. ${ }^{23}{ }^{24}$ For example, two task functional MRI (fMRI) studies focusing on male sexual arousal reported that, when compared with healthy controls, patients with pED manifested lower penile tumescence, more activity in the left superior parietal lobe, ventromedial prefrontal cortex, and posterior cingulate cortex, and altered intrinsic functional connectivity of the default mode network and salience network during visual erotic stimuli. ${ }^{23}{ }^{24}$ Resting-state fMRI studies also suggested that patients with pED not only displayed aberrant spontaneous activities at the right anterior insula but also showed abnormal connexion patterns between the right anterior insula and right dorsolateral prefrontal cortex as well as between the right anterior insula and right temporoparietal cortex. Furthermore, both the aberrant activities of the right anterior insula and the abnormal functional connexion between the right anterior insula and right temporoparietal cortex were positively correlated with participant scores on the International Index of Erectile Function (IIEF). ${ }^{20} 25$ On structural MRI, researchers found that, when compared with healthy controls, pED sufferers presented grey matter atrophy in some subcortical structures, including the amygdala and nucleus accumbens, and the atrophied degree of left nucleus accumbens showed a close correlation with decreased erectile function. ${ }^{26}$ Moreover, our previous study detected that patients with $\mathrm{pED}$ had significant microstructure alterations at the splenium of the corpus callosum and in multiple white matter regions. ${ }^{27}$

Based on these neuroimaging studies, we may easily conclude that pED is not only a genitourinary disease but also is associated with abnormal alterations in both brain structure and brain function. However, there is no integrated study summarising the scattered evidence of individual studies, and the central pathological alterations associated with pED remain unclear. Therefore, launching a rigorous systematic review to synthesise the hitherto existing studies is necessary to improve knowledge of the neurological underpinnings of $\mathrm{pED}$ and increase understanding of the role of the central nervous system in sexual activity.

\section{Objectives}

The objective of this systematic review is to integrate and assess the evidence of the impact of pED on the brain and to contribute a comprehensive summary of structural and functional brain alterations in patients with pED. This review also aims to synthesise correlations between the differences observed in some brain regions related to function or structure and the clinical characteristics of patients with $\mathrm{pED}$, such as behavioural and psychophysiological data and data obtained from disease-related scales, QoL scales and emotional scales.

\section{METHODS}

This protocol follows the Preferred Reporting Items for Systematic Review and Meta-Analysis Protocols 2015 statement $^{28}$ and has been registered at the PROSPERO International Prospective Register of Systematic Reviews of the University of York (registration number CRD42019117206).

\section{Eligibility criteria}

The inclusion and exclusion criteria of studies will be described as follows.

\section{Study types}

Case control studies, cohort studies and randomised controlled trials will be included only if the original neuroimaging data can be extracted. Case reports, narrative or systematic reviews, meta-analyses, letters and other secondhand studies will be excluded.

\section{Study design}

Neuroimaging studies centred on the differences between the brain structure, brain functional activity, and structural and functional connectivity of patients with pED and those of healthy controls will be included. Longitudinal studies focusing on the management of pED will be considered as long as the baseline neuroimaging data are reported. Both resting-state and task neuroimaging studies will be included, and no neuroimaging modality will be precluded. Any publication acquired data using multimodal neuroimaging techniques from the same participants will be collected separately in this review. ${ }^{29}$

\section{Participants}

Studies containing both patients with pED and parallel healthy controls will be considered for inclusion. The minimum sample size for inclusion will be restricted to 12 participants per group, according to previous studies. ${ }^{29-31}$ The race, age and disease conditions (drug-naïve or drug-invented) of participants will not be restricted in this review.

\section{Exposure}

Patients with pED should be diagnosed by comprehensive history taking, physical examination and even specific examinations according to the diagnostic guidelines of the European Association of Urology, ${ }^{32-34}$ the American Urological Association, ${ }^{35} 36$ or other authoritative organisations. ${ }^{37}$ Patients with organic ED or mixed ED or with other andrological or cardiovascular complications will be excluded. Some studies enrolling patients without clear discrimination of subtypes of ED will be considered after comprehensive full-text assessment or contact with the authors to identify the participants as patients with pED. 


\section{Comparators}

Participation of a parallel healthy control group in the study is required for inclusion in the current review. Healthy controls must have no prior diagnosis of ED at enrolment, and this must be verified by clinical examination during the study. Studies absenting from healthy controls will be excluded.

\section{Outcome measures}

The primary outcomes of the included studies should be functional and structural brain alterations of the patients with $\mathrm{pED}$. Brain structure outcomes are related to white matter microstructure, grey matter density or volume, or structural connectivity. Outcomes related to brain function include whole-brain or region-of-interest functional activity or functional connectivity (fMRI based on blood-oxygen-level-dependent signal or cerebral blood flow), brain molecular metabolism (positron emission tomography or single-photon emission CT); neurochemical activity (magnetic resonance spectroscopy); or brain electrical activity (electroencephalogram). The secondary outcomes of these studies may contain behavioural and psychophysiological data (such as genital responses and heart and respiratory rates) ${ }^{23} 24$; symptom-related scales (such as IIEF-5, ${ }^{38}$ Quality of Erection Questionnaire ${ }^{39}$ and the Erection Hardness Score ${ }^{40}$ ); QoL questionnaire (the Sexual Life Quality Questionnaire) ${ }^{41}$; or psychological assessment scales (such as the Self-Rating Anxiety Scale, ${ }^{42}$ the Self-Rating Depression Scale ${ }^{43}$ and the Brief Psychiatric Rating Scale ${ }^{44}$ ). Studies with only a primary outcome will also be included in this review.

\section{Report characteristics}

Peer-reviewed original studies will be included. Conference proceedings and unpublished theses will be excluded. Publication time will be restricted to prior to 1 October 2019 (the anticipated completion date of this review), and language will be restricted to English and Chinese.

\section{Searching strategy}

Electronic searching will be conducted in PubMed, EMBASE, Web of Science, China Biology Medicine Database and CNKI using medical subject headings (MeSH) terms. The searching strategies of PubMed (English) and CNKI (Chinese) are displayed in table 1 and will be replicated for the other electronic databases. Thereafter, the snowballing search strategy will be employed to find other eligible studies according to the reference lists of enrolled literature. In addition, the WHO International Clinical Trials Registry Platform will be searched for potential results.

\section{Selection process}

Covidence (https://www.covidence.org), the Cochrane Library-recommended online systematic review management system, will be used to manage literature. The initial searching results obtained following the above strategies will be uploaded to Covidence. After duplicates are
Table 1 Searching items for identifying articles in PubMed (English) and CNKI (Chinese)

\begin{tabular}{ll}
\hline & CNKI searching \\
PubMed searching strategy & strategy \\
\hline \#1 Erectile Dysfunction (MeSH & \#1 阳瘞(主题词) \\
Terms) & \#2 勃起功能障碍(主题词) \\
\#2 Impoten* (All Fields) & \#3 性功能障碍(主题词) \\
\#3 Erectile disturbance (All Fields) & \#4 ED(主题词) \\
\#4 Erectile disorder (All Fields) & \#5 \#1 OR \#2 OR\#3 OR \\
\#5 Sexual Dysfunction (MeSH & \#4 \\
Terms) & \#6 神经影像学(主题词) \\
\#6 Asynodia (All Fields) & \#7 功能磁共振(主题词) \\
\#7 Erection failure (All Fields) & \#8 磁共振成像(主题词) \\
\#8 Penile Erection (MeSH Terms) & \#9 MRI(主题词) \\
\#9 \#1 OR \#2 OR\#3 OR \#4 OR \#5 & \#10 PET(主题词) \\
OR \#6 OR \#7 OR \#8 & \#11 SPECT(主题词) \\
\#10 Neuroimaging (MeSH Terms) & \#12 EEG(主题词) \\
\#11 Functional Neuroimaging & \#13 MRS(主题词) \\
(MeSH Terms) & \#14 DTI(主题词) \\
\#12 Brain imaging (All Fields) & \#15 \#6 OR \#7 OR \#8 \\
\#13 MRI (MeSH Terms) & OR \#9 OR \#10 OR\#11 \\
\#14 Magnetic resonance* (MeSH & OR \#12 OR \#13 OR \#14 \\
Terms) & \\
\#15 MRI (All Fields) & \\
\#16 Tomography (MeSH Terms) & terms: \#5 AND \#15 \\
\#17 Positron Emission & \\
Tomography (MeSH Terms) & \\
\#18 Tomography, Emission- & \\
Computed, Single-Photon (MeSH & \\
Terms) & \\
\#19 PET (All Fields) & \\
\#20 PET-CT (All Fields) & \\
\#21 Single Photon Emission CT & \\
(MeSH Terms) & \\
\#22 SPECT (All Fields) & \\
\#23 Electroencephalography & \\
(MeSH Terms) & \\
\#24 EEG (All Fields) & \\
\#25 Magnetic Resonance & \\
Spectroscopy (MeSH Terms) & \\
\#26 MRS (All Fields) & \\
\#27 Diffusion Tensor Imaging & \\
(MeSH Terms) & \\
\#28 DTI (All Fields) & \\
\#29 \#10 OR\#11 OR \#12 OR \#13 & \\
\#30 Final search terms: \#9 AND & \\
\#29 & \\
\hline
\end{tabular}

removed, TY will screen the title and abstract to remove the obviously irrelevant records. Then two reviewers (TY and JX) will complete the abstract and full-text review in parallel. Any disagreement between TY and JX will be reconsidered by a third reviewer (ZL). In order to assess the reliability of the selection criteria and inter-rater agreement between the two reviewers, Cohen's kappa will be calculated at the parallel selection stage; a kappa coefficient $(\mathrm{k})$ over 0.75 will indicate high reliability. ${ }^{45}$ 
The record selection process will be reported using the Preferred Reporting Items for Systematic Reviews and Meta-Analyses flow diagram ${ }^{46}$ and elimination reasons for ineligible records will be reported in detail.

\section{Data collection}

The two independent reviewers (TY and JX) will doubly extract data using a standard data extraction spreadsheet in Excel. Again, any inconsistency between reviewers will be reconsidered and the result determined by ZL, the third reviewer.

The following information will be retrieved and extracted from each record.

- Publication information: title, first author, publishing time, country or region and funding support.

- Details of methodology: participants, sample size, diagnostic criteria, demographic characteristics (including age, handedness, ethnicity and education), imaging modalities, data analysis strategies, pED-related cognitive-behavioural models and clinical outcomes.

- Results: the significant altered cerebral regions (described by peak Montreal Neurological Institute $(\mathrm{MNI}) /$ Talairach coordinate, cluster size and statistical threshold); the value of clinical characteristics (behavioural and psychophysiological data, disease-related scales, QoL scales, emotional scales, etc); and the correlations between imaging data and clinical data.

Any missing information or questions about the above data will be settled by contacting the authors. If no clarification is provided after 4 weeks, the study will be included in the final analysis with the missing information marked.

\section{Outcomes and prioritisation}

The primary outcome of this review will be the significantly altered cerebral regions in patients with $\mathrm{PED}$ when compared with healthy controls. Due to the variety of analytical methods and great heterogeneity of the studies' statistical thresholds (e.g. voxel cluster size thresholds, statistical magnitudes, methods of correcting for multiple comparisons), it is unrealistic to set a uniform significance threshold. Therefore, the significance of results will be determined by the study authors' own criteria. ${ }^{47}$ Some neuroimaging studies also report results trending to significant or significant only before correction. ${ }^{47}$ For a more comprehensive view, these regions will be collected with special symbols in the qualitative synthesis. The secondary outcome of this review will be the associations between the altered cerebral structure and function and the clinical characteristics, which mainly include behavioural and psychophysiological data, disease-related scales, QoL scales, emotional scales and so on. The values of these clinical characteristics will be recorded and may be used to explain interstudy variability when necessary.

\section{Quality assessment}

There are no standardised criteria for quality assessment of neuroimaging studies. ${ }^{48}$ Authors of previous systematic reviews have always developed their own quality assessment tools based on existing tools (such as QUADAS-2 and the Newcastle-Ottawa Scale (NOS) $).{ }^{4850-54}$ However, because of the diverse study objectives, current assessment tools are not suitable for this review. Therefore, after referring to the $\mathrm{NOS}^{55}$ some published systematic reviews, ${ }^{48-50} 56$ and the Committee on Best Practices in Data Analysis and Sharing in Neuroimaging Using MRI ${ }^{57}$ (http://www.humanbrainmapping.org), a customised checklist is proposed for the current review. This checklist will be used to evaluate the quality of the included studies based on nine items (table 2). Each item is scored as 1 (Yes) or 0 (No or Don't know), and the summation of items generates an overall quality score (0-9 points). Each study's quality is defined as high (8-9 points), medium (5-7 points), or low ( $0-4$ points).

Quality assessment will be performed by a professional assessor (LL) who is experienced with quality assessment scoring and a nonprofessional assessor (RS) who has never engaged in quality assessment of systematic reviews. These two assessors will independently evaluate the enrolled studies based on the checklist; any discrepancy

Table 2 Checklist of quality assessment

$\begin{array}{lll}\text { Quality assessment categories } & \text { Yes No Don't know }\end{array}$

1. The study addressed an explicit question (theory-driven)

2. With sufficient sample size or used justified power calculation

\section{With clearly inclusion criteria and exclusion criteria of participants}

4. Controlled the important confounding factors such as age, handiness and education of participants

\section{With adequate quality control during data acquisition}

\section{Described the response rate in detail}

\section{Assessed outcomes with blinded or third-party assessors}

8. Used appropriate multiple testing correction in statistical modelling and inference

9. Reported detailed imaging results including MNI/Talairach coordinate, statistic magnitudes cluster sizes and statistical threshold 
will be reconsidered by the third reviewer (ZL). Again, the inter-rater agreement will be assessed by Cohen's kappa with $\mathrm{k}>0.75$ indicating high reliability.

\section{Data synthesis}

The collected data, including publication information, methodologies, and significant study findings, will be summarised in a table. Methodologies and neuroimaging results will then be pooled and described in detail. The total and average sample size, age range of participants and mean duration of patients of included studies will be calculated, and the cognitive-behavioural models, outcomes of behavioural and psychophysiological measurement, disease-related scales, QoL scales, and emotional scales will be summarised. A qualitative review will be performed to synthesise the structural and functional brain alterations and correlations between these alterations and the clinical characteristics of patients with pED. For more clear presentation, these findings will be integrated separately according to task or resting design and neuroimaging modality. If feasible (if 17 or more resting-state studies are included), ${ }^{58}$ an activation likelihood estimation meta-analysis ${ }^{59}{ }^{60}$ will be launched to quantitatively synthesise the differences in cerebral structure and function between patients with $\mathrm{pED}$ and healthy controls. Subgroup analyses will not be performed in this review. The strength of evidence of this review will be determined by the checklist described above.

\section{CONCLUSION}

Although neuroimaging studies have verified the existence of structural and functional brain alterations in patients with $\mathrm{pED}$, the scattered neuroimaging biomarkers of pED in individual studies have not yet been summarised. Therefore, this systematic review is launched, aiming to synthesise the central pathological characteristics and the associations between the altered cerebral structure and function and the clinical characteristics of pED. The current review will be the first to synthesise the neuroimaging evidence of pED in a systematic way, to include a meta-analysis of the findings, and to assess the quality of these neuroimaging studies. This work will provide a coherent synthesis of the recent neuroimaging studies on pED and improve knowledge of the neurological underpinnings of $\mathrm{pED}$.

\section{Patient and public involvement}

This is a protocol for systematic review. No patients and public were involved.

\section{Ethics and dissemination}

Ethical approval is not required as primary data will not be collected. This review will be published in a peer-reviewed journal and presented at conferences.

Contributors PZ was responsible for this study. TY, ZL and PZ conceived and designed the study. TY, ZL and JX participated in drafting the protocol and preparing the manuscript. LL, RS and FR provided feedback on the study design and protocol. All authors read and approved the final manuscript.

Funding This work is financially supported by the National Natural Science Foundation of China (№. 81774137).

Competing interests None declared.

Patient consent for publication Not required.

Provenance and peer review Not commissioned; externally peer reviewed.

Open access This is an open access article distributed in accordance with the Creative Commons Attribution Non Commercial (CC BY-NC 4.0) license, which permits others to distribute, remix, adapt, build upon this work non-commercially, and license their derivative works on different terms, provided the original work is properly cited, appropriate credit is given, any changes made indicated, and the use is non-commercial. See: http://creativecommons.org/licenses/by-nc/4.0/.

\section{REFERENCES}

1. Najari BB, Kashanian JA, Najari BB. Erectile dysfunction. JAMA 2016;316.

2. Muneer A, Kalsi J, Nazareth I, et al. Erectile dysfunction. BMJ 2014;348:9129.

3. Montorsi F, Adaikan G, Becher E, et al. Summary of the recommendations on sexual dysfunctions in men. $J$ Sex Med 2010;7:3572-88.

4. Rosen RC, Fisher WA, Eardley I, et al. The multinational men's attitudes to life events and sexuality (males) study: I. prevalence oSf erectile dysfunction and related health concerns in the general population. Curr Med Res Opin 2004;20:607-17.

5. Li H-J, Bai W-J, Dai Y-T, et al. An analysis of treatment preferences and sexual quality of life outcomes in female partners of Chinese men with erectile dysfunction. Asian J Androl 2016;18:773-9.

6. Yafi FA, Jenkins L, Albersen M, et al. Erectile dysfunction. Nat Rev Dis Primers 2016;2.

7. Boddi V, Corona G, Fisher AD, et al. "It takes two to tango": the relational domain in a cohort of subjects with erectile dysfunction (ED). J Sex Med 2012;9:3126-36.

8. Uddin SMI, Mirbolouk M, Dardari Z, et al. Erectile dysfunction as an independent predictor of future cardiovascular events. Circulation 2018;138:540-2.

9. Dong J-Y, Zhang Y-H, Qin L-Q. Erectile dysfunction and risk of cardiovascular disease: meta-analysis of prospective cohort studies. J Am Coll Cardiol 2011;58:1378-85.

10. Glina S, Cohen DJ, Vieira M. Diagnosis of erectile dysfunction. Curr Opin Psychiatry 2014;27:394-9.

11. Shamloul R, Ghanem H. Erectile dysfunction. Lancet 2013;381:153-65.

12. Rastrelli G, Maggi M. Erectile dysfunction in fit and healthy young men: psychological or pathological? Transl Androl Urol 2017;6:79-90.

13. Papagiannopoulos D, Nehra A, Khare N. Evaluation of young men with organic erectile dysfunction. Asian J Androl 2015;17:11-16.

14. Courtois F, Carrier S, Charvier K, et al. The control of male sexual responses. Curr Pharm Des 2013;19:4341-56.

15. Argiolas A, Rosaria Melis M. Neuromodulation of penile erection: an overview of the role of neurotransmitters and neuropeptides. Prog Neurobiol 1995;47:235-55.

16. Poeppl TB, Langguth B, Laird AR, et al. The functional neuroanatomy of male psychosexual and physiosexual arousal: a quantitative metaanalysis. Hum Brain Mapp 2014;35:1404-21.

17. Chen J, Chen $\mathrm{Y}, \mathrm{Gao} Q$, et al. Impaired Prefrontal-Amygdala pathway, self-reported emotion, and erection in psychogenic erectile dysfunction patients with normal nocturnal erection. Front Hum Neurosci 2018;12:157.

18. Chen J, Chen Y, Chen G, et al. Altered brain networks in psychogenic erectile dysfunction: a resting-state fMRI study. Andrology 2017;5:1073-81.

19. Winder K, Linker RA, Seifert F, et al. Insular multiple sclerosis lesions are associated with erectile dysfunction. J Neurol 2018;265:783-92.

20. Jin C, Guan M, Dong M, et al. Aberrant baseline brain activity in psychogenic erectile dysfunction patients: a resting state fMRI study. Brain Imaging Behav 2018;12:1393-404.

21. Chen J, Chen Y, Gao Q, et al. Brain structural network topological alterations of the left prefrontal and limbic cortex in psychogenic erectile dysfunction. Int $J$ Neurosci 2018;128:393-403.

22. Wang T, Liu B, Wu Z-J, et al. [Hypothalamus may be involved in psychogenic erectile dysfunction]. Zhonghua Nan Ke Xue 2008;14:602-5. 
23. Cera N, Di Pierro ED, Sepede G, et al. The role of left superior parietal lobe in male sexual behavior: dynamics of distinct components revealed by FMRI. J Sex Med 2012;9:1602-12.

24. Cera N, Di Pierro ED, Ferretti A, et al. Brain networks during free viewing of complex erotic movie: new insights on psychogenic erectile dysfunction. PLoS One 2014;9:e105336.

25. Wang $Y$, Dong $M$, Guan M, et al. Aberrant Insula-Centered functional connectivity in psychogenic erectile dysfunction patients: a resting-state fMRI study. Front Hum Neurosci 2017; $11: 221$

26. Cera N, Delli Pizzi S, Di Pierro ED, et al. Macrostructural alterations of subcortical grey matter in psychogenic erectile dysfunction. PLOS One 2012;7:e39118.

27. Zhang P, Liu J, Li G, et al. White matter microstructural changes in psychogenic erectile dysfunction patients. Andrology 2014;2:379-85.

28. Moher D, Shamseer L, Clarke M, et al. Preferred reporting items for systematic review and meta-analysis protocols (PRISMA-P) 2015 statement. Syst Rev 2015;4:1.

29. Lees B, Mewton L, Stapinski L, et al. Binge drinking in young people: protocol for a systematic review of neuropsychological, neurophysiological and neuroimaging studies. BMJ Open 2018;8:e023629.

30. Feldstein Ewing SW, Sakhardande A, Blakemore S-J. The effect of alcohol consumption on the adolescent brain: a systematic review of MRI and fMRI studies of alcohol-using youth. Neuroimage 2014;5:420-37.

31. Desmond JE, Glover GH. Estimating sample size in functional MRI (fMRI) neuroimaging studies: statistical power analyses. J Neurosci Methods 2002:118:115-28.

32. Wespes E, Amar E, Hatzichristou D, et al. Guidelines on erectile dysfunction. Eur Urol 2002;41:1-5.

33. Wespes E, Amar E, Hatzichristou D, et al. EAU guidelines on erectile dysfunction: an update. Eur Urol 2006;49:806-15.

34. Hatzimouratidis K, Amar E, Eardley I, et al. Guidelines on male sexual dysfunction: erectile dysfunction and premature ejaculation. Eur Urol 2010;57:804-14.

35. Montague DK, Jarow JP, Broderick GA, et al. Chapter 1: the management of erectile dysfunction: an AUA update. $J$ Urol 2005:174:230-9.

36. Burnett AL, Nehra A, Breau RH, et al. Erectile dysfunction: AUA guideline. J Urol 2018;200:633-41.

37. Ryu JK, Cho KS, Kim SJ, et al. Korean Society for sexual medicine and Andrology (KSSMA) guideline on erectile dysfunction. World $J$ Mens Health 2013;31:83-102.

38. Rosen RC, Riley A, Wagner G, et al. The International index of erectile function (IIEF): a multidimensional scale for assessment of erectile dysfunction. Urology 1997;49:822-30.

39. Porst $\mathrm{H}$, Gilbert $\mathrm{C}$, Collins $\mathrm{S}$, et al. Development and validation of the quality of erection questionnaire. J Sex Med 2007;4:372-81.

40. Mulhall JP, Goldstein I, Bushmakin AG, et al. Validation of the erection hardness score. J Sex Med 2007;4:1626-34.

41. Woodward JMB, Hass SL, Woodward PJ. Reliability and validity of the sexual life quality questionnaire (SLQQ). Qual Life Res 2002:11:365-77.

42. Zung WW. A rating instrument for anxiety disorders. Psychosomatics 1971;12:371-9.

43. Zung WW. A self-rating depression scale. Arch Gen Psychiatry $1965 ; 12: 63-70$.
44. Overall JE, Gorham DR. The brief psychiatric rating scale. Psychol Rep 1962;10:799-812.

45. McHugh ML. Interrater reliability: the kappa statistic. Biochemia Medica 2012;22:276-82.

46. Moher D, Liberati A, Tetzlaff J, et al. Preferred reporting items for systematic reviews and meta-analyses: the PRISMA statement. PLoS Med 2009;6:e1000097.

47. Fox KCR, Nijeboer S, Dixon ML, et al. Is meditation associated with altered brain structure? A systematic review and meta-analysis of morphometric neuroimaging in meditation practitioners. NeurosCi Biobehav Rev 2014;43:48-73.

48. Soiza RL, Sharma V, Ferguson K, et al. Neuroimaging studies of delirium: a systematic review. J Psychosom Res 2008;65:239-48.

49. Haggstrom L, Welschinger R, Caplan GA. Functional neuroimaging offers insights into delirium pathophysiology: a systematic review. Australas J Ageing 2017;36:186-92.

50. Lavagnino L, Arnone D, Cao B, et al. Inhibitory control in obesity and binge eating disorder: a systematic review and meta-analysis of neurocognitive and neuroimaging studies. Neurosci Biobehav Rev 2016:68:714-26.

51. Sumner PJ, Bell IH, Rossell SL. A systematic review of task-based functional neuroimaging studies investigating language, semantic and executive processes in thought disorder. Neurosci Biobehav Rev 2018;94:59-75

52. McCrea M, Meier T, Huber D, et al. Role of advanced neuroimaging, fluid biomarkers and genetic testing in the assessment of sportrelated concussion: a systematic review. Br J Sports Med 2017;51:919-29.

53. Ailion AS, Hortman K, King TZ. Childhood brain tumors: a systematic review of the structural neuroimaging literature. Neuropsychol Rev 2017;27:220-44.

54. Cagnie B, Coppieters I, Denecker S, et al. Central sensitization in fibromyalgia? A systematic review on structural and functional brain MRI. Semin Arthritis Rheum 2014:44:68-75.

55. Wells GA, Shea B, O'Connell D, et al. The Newcastle-Ottawa scale (NOS) for assessing the quality of nonrandomised studies in meta-analyses. Secondary the Newcastle-Ottawa scale (NOS) for assessing the quality of nonrandomised studies in meta-analyses, 2013. Available: http://www.ohri.ca/programs/clinical_epidemiology/ oxford.asp

56. Wilson PH, Smits-Engelsman B, Caeyenberghs K, et al. Cognitive and neuroimaging findings in developmental coordination disorder: new insights from a systematic review of recent research. Dev Med Child Neurol 2017;59:1117-29.

57. Nichols TE, Das S, Eickhoff SB, et al. Best practices in data analysis and sharing in neuroimaging using MRI. Nat Neurosci 2017;20:299-303.

58. Eickhoff SB, Nichols TE, Laird AR, et al. Behavior, sensitivity, and power of activation likelihood estimation characterized by massive empirical simulation. Neuroimage 2016;137:70-85.

59. Eickhoff SB, Laird AR, Grefkes C, et al. Coordinate-based activation likelihood estimation meta-analysis of neuroimaging data: a randomeffects approach based on empirical estimates of spatial uncertainty. Hum Brain Mapp 2009;30:2907-26.

60. Turkeltaub PE, Eden GF, Jones KM, et al. Meta-analysis of the functional neuroanatomy of single-word reading: method and validation. Neuroimage 2002;16:765-80. 